\title{
Rate and timing dependent plasticity in a biophysical model of synaptic plasticity
} Arvind Kumar*, Luk Chong Yeung and Mayank R Mehta*

\author{
Address: Department of Neuroscience Box 1953, Brown University, Providence, RI 02912, USA \\ Email: Arvind Kumar* - arvind_kumar@brown.edu; Mayank R Mehta* - mayank_mehta@brown.edu \\ * Corresponding authors
}

from Sixteenth Annual Computational Neuroscience Meeting: CNS*2007

Toronto, Canada. 7-12 July 2007

Published: 6 July 2007

BMC Neuroscience 2007, 8(Suppl 2):P85 doi: I0.1 I86/I47I-2202-8-S2-P85

C 2007 Kumar et al; licensee BioMed Central Ltd.

Activity dependent synaptic plasticity is typically induced by delivering a large number $(\sim 1000)$ of highly regular electrical stimuli, designed to saturate the synaptic strengths. However, natural spike trains typically contain a small number of spikes with variable regularity. We investigate how spike patterns with physiologically realistic spike counts generate plasticity using a standard, biophysical model of calcium-dependent plasticity. We find that for physiological spike patterns, there exists a resonant frequency (fmax) that induces maximal firing rate dependent potentiation and periodic stimuli produce substantially more plasticity than aperiodic ones. Frequency dependent facilitation (depression) of the synapses does not affect the fmax but increases (decreases) the amount of plasticity; relative change in the amount of plasticity varies with number of spikes. The model combines the rate dependent plasticity with spike timing dependent plasticity (STDP) and shows that the direction of STDP depends both on the number of postsynaptic spikes that a single presynaptic spike is paired with and on the frequency of postsynaptic discharge. We discuss experimental tests of these results, and their functional significance for learning under natural, dynamical conditions.

\section{Acknowledgements}

This work was supported by the Rhode Island foundation, the Salomon foundation, NIH/CRCNS, a young investigator award from the NARSAD foundation and an early career award from NSF. 